\title{
Squamous Cell Lung Carcinoma Presenting With Refractory Status Epilepticus: A Case Report of Paraneoplastic Limbic Encephalitis
}

\author{
Killian Hurley MB BCh BAO PhD, Malcolm Herron MB BCh BAO, \\ Sean McDermott MB BCh BAO, Terence Farrell MB BCh BAO, and \\ Deirdre O'Riordan MB BCh BAO
}

\begin{abstract}
Lung cancer-associated paraneoplastic syndromes affecting the central nervous system present significant diagnostic and treatment challenges. In this case, the patient presented with personality change, cognitive impairment, complex partial seizures, ataxia, dyspraxia, and dysphasia. Shortly after admission, the patient suffered refractory generalized tonic-clonic seizures and a decreased level of consciousness and required intubation, ventilation, and admission to the ICU. He was subsequently diagnosed with paraneoplastic limbic encephalitis based on recognized criteria, including a compatible clinical picture, elevated protein content in his cerebrospinal fluid with negative cytology, a positive positron emission tomography-computed tomography scan showing a right upper lobe tumor, and the exclusion of other neuro-oncological complications. Histopathology confirmed the tissue diagnosis as squamous cell cancer. Initial immunotherapy with steroids and intravenous immunoglobulin and subsequent lobectomy and adjuvant chemotherapy were partially successful, leading to partial resolution of his cognitive impairment. This report highlights the diagnostic and therapeutic challenges of lung-related paraneoplastic syndromes. In addition, it illustrates the poor outcomes for patients and identifies squamous cell cancer as an extremely rare cause of paraneoplastic limbic encephalitis. Key words: lung cancer; squamous cell carcinoma; seizures; paraneoplastic syndrome; limbic encephalitis; intensive care unit. [Respir Care 2015;60(8):e144-e147. (C) 2015 Daedalus Enterprises]
\end{abstract}

\section{Introduction}

Paraneoplastic syndromes are diseases that are the consequence of cancer but are not due to the local presence of cancer cells. ${ }^{1}$ Instead, tumor cells are thought to express tissue-specific antigens, such as neuronal proteins. These can trigger an antitumor immune response that can attack the normal tissue expressing that (eg, neuronal) protein.

Drs Hurley, Herron, McDermott, and O'Riordan are affiliated with the Department of Internal Medicine, and Dr Farrell is affiliated with the Department of Radiology, St. James's Hospital, Dublin, Ireland.

Drs Hurley and Herron are co-first authors.

The authors have disclosed no conflicts of interest.

Correspondence: Killian Hurley $\mathrm{MB}$ BCh BAO PhD, Department of Internal Medicine, St. James's Hospital, Dublin 8, Ireland. E-mail: killian_hurley@yahoo.com.

DOI: $10.4187 /$ respcare.03879
Lung cancer-associated paraneoplastic syndromes affecting the central nervous system present significant diagnostic and treatment challenges. However, substantial advances in the investigation of these syndromes, such as detection of anti-neuronal antibodies, have improved recognition of known syndromes, and the use of computer tomography (CT) and positron emission tomography (PET)-CT to identify the associated tumors has led to early diagnosis of these complex disorders. In this case report, we describe a case of refractory seizures requiring intubation, ventilation, and a complex ICU stay caused by paraneoplastic limbic encephalitis (PLE) originating from squamous cell lung carcinoma.

\section{Case Report}

A 64-y-old man presented to the accident and emergency department of an inner city university teaching hospital with acute confusion, complex partial seizures, and a change in his personality over the preceding 2 weeks. At the time of admission, his family described a history of 
Table 1. Serum Anti-Neuronal Antibodies Tested

\begin{tabular}{lc}
\hline \hline \multicolumn{1}{c}{ Antibody } & Result \\
\hline Anti-Ri antibody & Negative \\
Anti-Yo antibody & Negative \\
Anti-Hu antibody & Negative \\
AMPA1 antibody & Negative \\
AMPA2 antibody & Negative \\
Anti-NMDA receptor antibody & Negative \\
Anti-Ma1 antibody & Negative \\
Anti-Ma2 antibody & Negative \\
Anti-CV/CRMP5 antibody & Negative \\
Anti-amphiphysin antibody & Negative \\
Potassium channel antibody & Negative \\
Anti-GABA antibody & Negative \\
& \\
\hline NMDA $=N$-methyl-D-aspartate & \\
GABA $=$ gamma-aminobutyric acid & \\
\hline
\end{tabular}

falls, unsteady gait, slurred speech, and difficulties in finding words. They also described a number of episodes of staring blankly for $>30 \mathrm{~s}$ without a response over the previous 2 weeks. The patient described a new tremor in his hands and dyspraxia. His background was significant for hypertension, hypercholesterolemia, a 60 pack-year smoking history, and surgical repair of a hydrocele. He had a distant history of alcohol excess but at the time of admission was drinking $\sim 10$ standard drinks/week. His medications included captopril and amlodipine.

On physical examination, there was an upper limb intention tremor, horizontal gaze nystagmus, and ataxia, but the remainder of the neurological examination was otherwise normal. Neurocognitive testing with the Montreal Cognitive Assessment revealed a score of 10/30 on admission to the hospital. Initial blood work and chest radiographs performed in the emergency department and repeated later on the ward and in the ICU were reported as normal. He subsequently had a brain CT that showed normal brain parenchyma and ventricular size with no acute hemorrhage or infarction. Magnetic resonance imaging (MRI) of the brain showed mild global atrophy with normal brain parenchyma, and diffusion-weighted imaging was normal with no evidence of an acute infarct.

An initial evaluation of the cerebrospinal fluid performed by lumber puncture showed a white blood cell count of $<1 \times 10^{3}$ cells/ $\mu \mathrm{L}$, a protein level of $111 \mathrm{mg} / \mathrm{dL}$ (normal range of $15-45 \mathrm{mg} / \mathrm{dL})$, and a glucose level of $58 \mathrm{mg} / \mathrm{dL}$ ( $\sim 60 \%$ of serum). The sample was also examined by Gram staining (which was found to be negative), culture, sensitivity, and polymerase chain reaction for herpes simplex virus types 1 and 2, varicella-zoster virus, and Enterovirus. Paraneoplastic antibodies were requested from the serum at this time (Table 1) and were all found to be negative, as was a human immunodeficiency virus screen. The patient was treated empirically for encephalitis with intravenous acyclovir until all viral polymerase chain reaction analyses were confirmed to be negative. A diagnosis of Wernicke encephalopathy was also considered given the patient's presentation and significant but distant excess alcohol history, and the patient was treated with high-dose parenteral thiamine twice a day for $3 \mathrm{~d}$ without clinical improvement.

At $2 \mathrm{~d}$ after admission, the patient deteriorated, characterized by multiple unresponsive episodes, myoclonic jerks, erratic eye movements, and incoherent muttering. He developed generalized tonic-clonic seizures refractory to multiple antiepileptic medications, including midazolam, valproate, levetiracetam, and phenytoin. Subsequently, he was transferred to the ICU, where he was intubated, ventilated, and sedated with midazolam, propofol, and remifentanil due to his decreased level of consciousness and refractory status epilepticus. At this time, a working diagnosis of paraneoplastic syndrome was made based on his clinical presentation, sterile cerebrospinal fluid with high protein, and lack of an isolated infective organism. He was therefore begun on a trial of high-dose intravenous methylprednisolone and intravenous immunoglobulin. He continued to have convulsions, focal jerking, automatism, and abnormal posturing. A continuous electroencephalogram showed persistent seizure activity, which eventually improved on day 4 of intravenous steroids and immunoglobulin. He was weaned from sedation, which was discontinued on day 5 of his ICU stay. The patient was extubated and discharged to a medical ward on sodium valproate, levetiracetam, and phenytoin, having spent $10 \mathrm{~d}$ in the ICU.

During further investigation to determine the cause of the patient's seizures, thorax, abdomen, and pelvis CTs were performed and identified a $2.9-\mathrm{cm}$ nodule in the posterior segment of the right upper lobe abutting the oblique fissure. This nodule was not appreciated on the initial chest radiograph or on follow-up films in the ICU. A subsequent PET-CT (Fig. 1) revealed a fluorodeoxyglucose-avid mass in the right upper lobe with no lymph node involvement. Endobronchial biopsy secured the histological diagnosis of squamous cell carcinoma (Fig. 2). After extensive workup, including pulmonary function testing and echocardiography, it was concluded at the hospital's lung cancer multidisciplinary meeting that the patient had stage T1b/N0/M0 or stage IA non-small cell lung cancer, and a right upper lobectomy was subsequently performed. After resection of the primary, the oblique fissure was found not to be involved, and a single ipsilateral hilar node was found to be positive for cancer, giving a revised stage of $\mathrm{T} 1 \mathrm{~b} / \mathrm{N} 1 / \mathrm{M} 0$ or stage IIA. Therefore, adjuvant chemotherapy with cisplatin/vinorelbine at days 1 and 8 every 3 weeks for 4 cycles was advised. Repeated neurocognitive testing immediately postoperatively with the Montreal Cognitive Assessment revealed a score of 20/30, double 

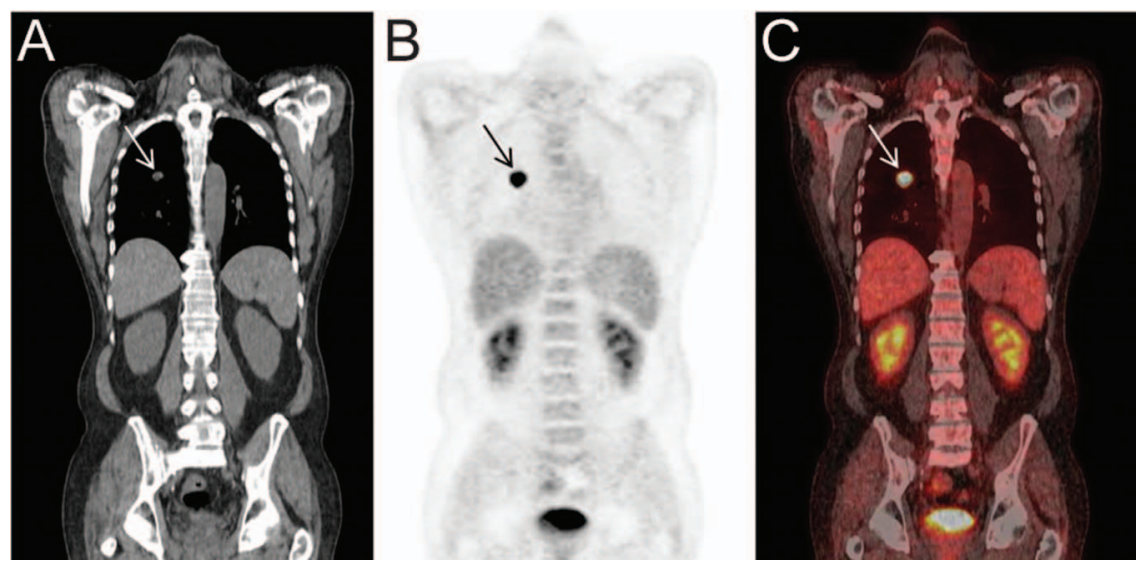

Fig. 1. Computed tomography (CT) and positron emission tomography (PET) of the right upper lobe tumor. A: Coronal CT. B: PET. C: Fused PET-CT images showing increased $\left[{ }^{18} \mathrm{~F}\right]$ fluorodeoxyglucose uptake in the right upper lobe with a significant standardized uptake value of 7.5 .

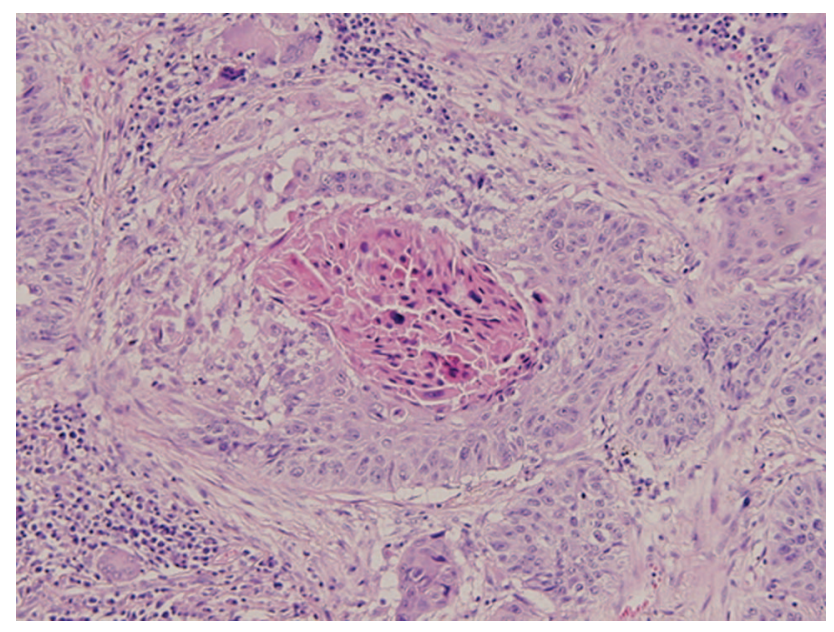

Fig. 2. Histological appearance of the endobronchial biopsy of the tumor in the right upper lobe. The $\times 20$ view of cells stained with hematoxylin and eosin shows nests of keratinizing squamous cells, confirming the presence of squamous cell cancer.

his preoperative score, and he went on to receive adjuvant chemotherapy as an out-patient, having been discharged home to his family. Two months after the lobectomy, the patient was well and seizure-free and had been weaned off his antiepileptic medications and steroids. Four months after surgery, the patient again deteriorated, with decreased cognitive function and absence-like seizures. Neurological examination, contrast-enhanced brain MRI, and thoracic CT did not reveal cerebral metastases or disease progression, so steroids, intravenous immunoglobulin, and antiepileptic medications were recommenced for relapse of paraneoplastic syndrome. Two months later, although being seizure-free, he had not regained his premorbid cognitive function and had marked behavioral and personality changes despite sustained immunotherapy.

\section{Discussion}

Paraneoplastic syndrome characterized by seizures, personality changes, psychological irritability, depression, and memory loss is extremely rare and was first described as PLE by Corsellis et $\mathrm{al}^{2}$ in 1968. Since then, a small number of cases have been described in the literature, mainly in the form of isolated case reports and small case series. The most comprehensive study so far was conducted by Dalmau and colleagues, ${ }^{3}$ who examined the clinical and laboratory data of 50 subjects in a single-center study. In this study, the diagnosis of PLE required neuropathological examination or the presence of all 4 of the following criteria: (1) a compatible clinical picture; (2) an interval of $<4$ y between the development of neurological symptoms and tumor diagnosis; (3) exclusion of other neuro-oncological complications; and (4) at least one of the following: cerebrospinal fluid with inflammatory changes but negative cytology, MRI demonstrating temporal lobe abnormalities, or electroencephalogram showing epileptic activity in the temporal lobes. Furthermore, the authors found that PLE was associated with lung cancer (predominantly small cell lung cancer) in 50\% of the cases examined and that only $60 \%$ of the subjects had positive anti-neuronal antibodies.

In this case, the diagnosis of PLE was confirmed by a compatible clinical picture, a positive PET-CT scan showing a right upper lobe tumor confirmed as squamous cell cancer by histopathology within weeks of the onset of symptoms, the exclusion of other neurological complications of cancer, and elevated cerebrospinal fluid protein content with negative cytology as per the above diagnostic criteria. ${ }^{3}$ To our knowledge, this is only the second case of PLE associated with squamous cell lung carcinoma reported in the literature. ${ }^{4}$ In most case series, between 54 and $80 \%$ of lung cancer-associated PLE is due to small 
cell lung cancer, is anti-Hu antibody-positive (the most well recognized anti-neuronal antibody ${ }^{5}$, and has a poor outcome. . $^{3,6,67}$ It is interesting to note that anti-neuronal antibodies such $\mathrm{Hu}$ were not identified in this case, and indeed, we may postulate that the culprit autoantibody associated with squamous cell cancer has yet to be identified.

This case raises an interesting point about the pathogenesis of PLE in lung cancer, as it occurred in an individual with non-small cell lung cancer, which, unlike small cell lung cancer, is amenable to curative surgery. Therefore, one might hypothesize that surgery may also ameliorate paraneoplastic symptoms by removing the primary instigator of antibody formation. However, little is known about the persistence of paraneoplastic symptoms postoperatively. Indeed, this patient initially responded to immunotherapy with high-dose steroids, immunoglobulin G, and subsequent surgery with a marked improvement in neurophysiological testing. Despite the early success of therapy, symptoms returned after the patient was weaned from steroids and immunoglobulin and were only finally controlled after the reinstitution of immunotherapy postoperatively, indicating that removing the primary cancer in this patient did not resolve his paraneoplastic symptoms. It is possible, however, that the patient had minimally metastatic cancer, as one node was positive at the time of surgery, and this finding may explain the persistence of his symptoms. Treatment-responsive limbic encephalitis has been described in patients who do not possess the classical autoantibodies but are characterized by antibodies to the neuropil of the hippocampus or cerebellum using a novel experimental immunohistochemical technique. ${ }^{8}$ However, overall neurological recovery in patients with PLE is rare in the reported literature, ${ }^{6,9}$ and treatment with immunotherapy has yielded disappointing results. ${ }^{10}$ Our patient made great improvements in his cognitive impairment after immunotherapy and surgery; however, his impairment it was still significant enough to be debilitating.

In conclusion, this report highlights the diagnostic and therapeutic challenges of lung cancer-related paraneoplastic syndromes. The severity of the patient's presentation with refractory status epilepticus was highly unusual for PLE, and initial screening with anti-neuronal antibodies and chest radiographs did not identify an obvious malignancy. Indeed, thoracic CT and PET-CT were needed to identify the source, and extensive anti-neuronal antibody screening was negative. Importantly, squamous cell cancer has only once been reported to cause PLE. Initial immunotherapy with subsequent surgery and chemotherapy for our patient's primary lung cancer were partially successful, but ultimately, his cognitive impairment did not completely resolve, highlighting the devastating effect of this difficult-to-diagnose condition.

\section{ACKNOWLEDGMENTS}

We acknowledge Dr Siobhán Nicholson (consultant pathologist, St James's Hospital, Dublin, Ireland) for supplying the histological image and description of the tumor resected from the right upper lobe.

\section{REFERENCES}

1. Darnell RB, Posner JB. Paraneoplastic syndromes involving the nervous system. N Engl J Med 2003;349(16):1543-1554.

2. Corsellis JA, Goldberg GJ, Norton AR. "Limbic encephalitis" and its association with carcinoma. Brain 1968;91(3):481-496.

3. Gultekin SH, Rosenfeld MR, Voltz R, Eichen J, Posner JB, Dalmau J. Paraneoplastic limbic encephalitis: neurological symptoms, immunological findings and tumour association in 50 patients. Brain 2000; 123(Pt 7):1481-1494.

4. Lawn ND, Westmoreland BF, Kiely MJ, Lennon VA, Vernino S. Clinical, magnetic resonance imaging, and electroencephalographic findings in paraneoplastic limbic encephalitis. Mayo Clinic Proc 2003;78(11):1363-1368.

5. Dalmau J, Rosenfeld MR. Paraneoplastic syndromes of the CNS. Lancet Neurol 2008;7(4):327-340.

6. Dalmau J, Graus F, Rosenblum MK, Posner JB. Anti-Hu-associated paraneoplastic encephalomyelitis/sensory neuronopathy. A clinical study of 71 patients. Medicine 1992;71(2):59-72.

7. Graus F, Keime-Guibert F, Reñé R, Benyahia B, Ribalta T, Ascaso $\mathrm{C}$, et al. Anti-Hu-associated paraneoplastic encephalomyelitis: analysis of 200 patients. Brain 2001;124(Pt 6):1138-1148.

8. Ances BM, Vitaliani R, Taylor RA, Liebeskind DS, Voloschin A, Houghton DJ, et al. Treatment-responsive limbic encephalitis identified by neuropil antibodies: MRI and PET correlates. Brain 2005; 128(Pt 8):1764-1777.

9. Alamowitch S, Graus F, Uchuya M, Reñé R, Bescansa E, Delattre JY. Limbic encephalitis and small cell lung cancer. Clinical and immunological features. Brain 1997;120(Pt 6):923-928.

10. Sillevis Smitt P, Grefkens J, de Leeuw B, van den Bent M, van Putten W, Hooijkaas H, Vecht C. Survival and outcome in 73 anti$\mathrm{Hu}$ positive patients with paraneoplastic encephalomyelitis/sensory neuronopathy. J Neurol 2002;249(6):745-753. 